\title{
REVERSED DETERMINANTAL INEQUALITIES FOR ACCRETIVE-DISSIPATIVE MATRICES
}

\author{
MINGHUA LIN
}

Abstract. A matrix $A \in M_{n}(\mathbf{C})$ is said to be accretive-dissipative if, in its Toeplitz decomposition $A=B+i C, B=B^{*}, C=C^{*}$, both matrices $B$ and $C$ are positive definite. Let $A=\left[\begin{array}{ll}A_{11} & A_{12} \\ A_{21} & A_{22}\end{array}\right]$ be an accretive-dissipative matrix, $k$ and $l$ be the orders of $A_{11}$ and $A_{22}$, respectively, and let $m=\min \{k, l\}$. It is proved

$$
|\operatorname{det} A| \geqslant \frac{(4 \kappa)^{m}}{(1+\kappa)^{2 m}}\left|\operatorname{det} A_{11}\right|\left|\operatorname{det} A_{22}\right|,
$$

where $\kappa$ is the maximum of the condition numbers of $B$ and $C$.

Mathematics subject classification (2010): 15A45, 15A15.

Keywords and phrases: determinantal inequality, accretive-dissipative matrix, Schur complement.

\section{REFERENCES}

[1] D. J. Clements, H. K. Wimmer, Monotonicity of the optimal cost in the discrete-time regulator problem and Schur complements, Automatica 37 (2001), 1779-1786.

[2] A. George, Kh. D. Ikramov, On the properties of accretive-dissipative matrices, Math. Notes 77 (2005), 767-776.

[3] Kh. D. Ikramov, Determinantal inequalities for accretive-dissipative matrices, J. Math. Sci. (N. Y.) 121 (2004), 2458-2464.

[4] R. A. Horn And C. R. Johnson, Matrix Analysis, Cambridge University Press, London, 1985.

[5] F. ZHANG, Equivalence of the Wielandt inequality and the Kantorovich inequality, Linear Multilinear Algebra 48 (2001), 275-279. 